\section{GW23-e0571 ASSOCIATION OF INDUCED HEART RATE TURBULENCE WITH SEVERITY OF CORONARY ARTERY LESIONS IN CORONARY HEART DISEASE}

doi:10.1136/heartjnl-2012-302920n.23

Zhou Aiqin, Liao Wei, Zhong Yiming, Zhou Aiqin. Department of Cardiology, The First Affiliated Hospital of Gannan University, Ganzhou 341000, China

Objectives Impaired heart rate turbulence (HRT) denotes abnormalities in cardiac autonomic function. We aimed to assess the correlation between coronary artery lesion and HRT induced by programmed electrical stimulation.

Methods From January 2008 to January 2009, totally 113 patients (65 males and 48 females) who underwent coronary angiography were induced by programmed electrical stimulation and divided into four groups according to the number of coronary artery damage: normal control group $(n=28)$, single vessel damage group $(n=36)$, double vessels damage group $(n=26)$ and many vessels damage group $(n=23)$. The mean age of patients was $(60.7 \pm 11.3)$ years (ranged from 45 to 74 years). After completion of coronary angiography, an extrastimulus was delivered from the right ventricular apex after 20 sinus beats with a V-S2 coupling interval decremented by 20 to $30 \mathrm{~ms}$ until refractoriness was reached. It was completed respectively for the measurement of heart rate, turbulence onset (TO), turbulence slope (TS) and Gemini's score of coronary stenosis.

Results No serious complication were found during the programmed electrical stimulation, such as death and malignant ventricular arrhythmias. No significant differences were found in gender, age, blood pressure and heart rate. Compared with control group, TO was significantly higher but TS was significantly lower in coronary heart disease groups $(p<0.05$ or $p<0.01)$. With the increasing severity of coronary heart disease, TO level was increased but TS level was decreased $(p<0.05$ or $p<0.01)$. TS was negatively correlated with Gemini's score $(r=-0.245, p=0.009)$ but TO was positively correlated with it $(r=0.288, p=0.002)$. Balanced the influential factors such as age, gender, blood pressure and heart rate, TO and TS still had significantly correlated with Gemini's score $(p<0.01)$. Multiple regression analysis showed that Gemini's score was independent factor to influent TO and TS level.

Conclusions Our data show that it is safe to measure induced HRT after coronary angiography in patients with coronary heart disease. The abnormal parameters of HRT have close relationship with the severity of coronary artery stenosis. 